\title{
When CPAP is stopped: what are the "on switches" of sleep apnoea?
}

\author{
Kingman P. Strohl ${ }^{1}$ and Andrew Wellman ${ }^{2}$ \\ Affiliations: 'Case Medical Center and Louis Stokes DVA Medical Center, Case Western Reserve University, \\ Cleveland, $\mathrm{OH}$, and ${ }^{2}$ Brigham and Women's Hospital, Harvard Medical School, Boston, MA, USA.
}

Correspondence: K.P. Strohl, 111j(w) VAMC, 10701 East Boulevard, Cleveland, OH 44106, USA.

E-mail: Kingman.Strohldva.gov

O

@ERSpublications

Stopping CPAP could be used as a model for uncovering the reestablishment of OSA http://ow.ly/tvbJP

Adult sleep apnoea is considered a relatively common and chronic disease, with the defining features of sleep disordered breathing (apnoeas, hypopneas and respiratory effort-related arousals) leading to its consequences. The physiological sequelae include a fall in oxygen and rise in carbon dioxide, changes in intrathoracic pressure and cardiac performance, and/or arousals from sleep [1]. Morbidity and mortality occur due to daytime inattention and sleepiness (leading to impaired quality of life with drowsiness, and fall-asleep crashes and accidents), metabolic syndrome [2] and cardiovascular disease (hypertension, stroke, arrhythmia, coronary artery disease, etc.) [3, 4], with some recent evidence that apnoeas might confer a greater risk of cancer or cancer progression $[5,6]$. Yet it is widely accepted that there is imprecision in the links between events in sleep and these adverse outcomes, so that determining an individual's prognosis and need for immediate therapy is an uncertain art.

Obstructive sleep apnoea hypopnoea syndrome (OSAHS) is defined by symptoms of unrefreshing and disturbed sleep, loud snorts and snoring, and a certain number of events (usually more than five per hour) in a summary value called the apnoea-hypopnea index, and is relieved of symptoms when it is treated most directly by tracheostomy [7]. Continuous positive airway pressure (CPAP) has replaced tracheostomy as a therapy to reduce sleepiness and improve quality of life [8].

In this issue of the European Respiratory Journal, Rossi et al. [9] report that about a third of CPAP-treated obstructive sleep apnoea (OSA) patients do not experience recurrence of oxygen desaturations after four nights and $\sim 10 \%$ do not experience recurrence following 2 weeks of intentional CPAP withdrawal. All had been treated for $>2$ years. As the authors freely admit, this is a paper not without flaws, with a convenient design, outcome measures based on portable monitoring, lack of generalisation to all patients with sleep apnoea, and inability to place the findings within the broader categories of decision making and clinical management. The entry point for the patients is not very clear in terms of meeting the definition of a syndrome, other than meeting a measure during an in-hospital respiratory polygraphy: an oxygen desaturation index (ODI) using a $\geqslant 4 \%$ dip of $>10$ events per hour during sleep. There are no details of event number in rapid eye movement (REM) versus non-REM sleep, or in supine versus nonsupine posture. Note that the ODI as measured by ambulatory nocturnal pulse oximetry and, therefore, positional and sleep stage dependence would not be captured. Nevertheless, unlike the observations of remission of OSAHS with bariatric surgery (obesity confers a seven-fold increase in risk), there is a lack of obvious mitigation of risk by CPAP over time of the sort that most clinicians would have said "I could have predicted this."

One of us (K.P. Strohl) helped write articles describing the clinical understanding of sleep apnoea and the intent of different therapies to address suspected anatomical and neural factors in sleep disordered

Received: Jan 062014 | Accepted: Jan 142014

Conflict of interest: Disclosures can be found alongside the online version of this article at www.erj.ersjournals.com

Copyright (C)ER 2014 
breathing, and the elements of clinical risk gleaned in the examination of the patient $[7,10]$. Since then, the field has moved to more fundamental concerns $[1,11,12]$. Over the last few years, the other author (A. Wellman) and his group have developed a process of separating and measuring features during sleep that correlate with the expression of recurrent apnoeas in an individual patient $[13,14]$. These features include the critical closing pressure of the upper airway (Pcrit), the recruitment or gain of function of muscles that keep the airway patent, the threshold for arousal from sleep, and the tendency for a disturbance to "set up" the person for another apnoea (loop gain).

The article by ROSSI et al. [9] gives us the opportunity to consider how OSAHS could redevelop upon withdrawal of therapy. To move the discussion forward, we will stipulate that the patients who are described in this report had a bona fide clinical condition that, in the opinion of the sleep specialists, required direct therapy, and that CPAP treatment was successful and used at least most of the time by all patients. Furthermore, we will ignore the potential impact of sleep stage and position. Are there any clues as to which of these patients might not need CPAP 2 years later, for $\geqslant 2$ weeks?

From the dataset, moving $P$ crit to a more negative level might get you a long way in terms of understanding the responses of the 36 patients who still had an ODI $<10$ at withdrawal day 4 versus those who did not. The hypothesis of the study was based on reports of various changes in the upper airway (inflammation, oedema, trauma to tissues, etc.) in patients with OSA and the assumption that CPAP reduced the palatal "clatter and bang" of untreated OSA [1]. As a longer time even beyond the minimum of 2 years was associated with a higher ODI on withdrawal at 4 days, it may be that longer CPAP is better. Other significant factors found in a multivariable analysis were neck circumference and current smoking, which might reflect mass effects on the airway and some beneficial effect of smoking (although in a univariate model, current smoking was not significant), respectively [7]. Without direct measures of Pcrit, we will never know. This permits us the opportunity for rampant speculation on the other features.

Recruitment of muscles is a reflex mechanism for recovery of upper airway patency through direct and indirect actions on size and/or stiffness. One line of thought suggests that impaired recruitment beyond that occurring with sleep is the result of afferent deficits or efferent output [15-17]. Another is that there is an inherent gain of recruitment [18]. Furthermore, muscle efferent output must be translated into mechanical changes in the airway, which may be more difficult with trauma and oedema [19]. All might be improved by long-term CPAP but, again, how this explains the differences among individuals in the tendency to relapse or remit for a week or so without CPAP is unclear.

Arousal threshold will tend to decrease with treatment of OSA (patients will wake up more easily) [20, 21], which would tend to increase the propensity to respiratory effort-related arousals, i.e. shorter events with less desaturation. This would not be detected in the oximetry studies at a $4 \%$ threshold for a dip, at least directly. Arousals are a mechanism that both shortens events, and rapidly and suddenly increases "gain". It is a longer time to arousal that is thought to increase the chance of a person to increase drive, improve ventilation, maintain oxygenation and reduce arousals during sleep [20, 22]. These patients might not be suspected of having achieved deeper sleep or to have slept "better", whatever that means. A measure of this process can be obtained using measures of flow and arousals, and might provide clues as to these mechanisms of apnoea return or mitigation in the setting of CPAP withdrawal.

We turn now to loop gain, which is the propensity of the respiratory feedback control system to oscillate. A high loop gain here means the relative inability of a person's control system to return to steady breathing after a disturbance like an apnoea and a number of studies indicate that high loop gain is a risk factor for OSA. One property of loop gain is related to the effectors for ventilation (nerves, muscles and the chest wall) and another due to the response organised to the disturbance. We know that oxygen [23] can decrease loop gain and mitigate events in a proportion of patients, and common drugs like indomethacin can alter loop gain and increase events [24]. CPAP itself will lower loop gain [25]. A major component of loop gain, namely the ventilatory response to carbon dioxide, decreases (ventilation becomes more stable and less oscillatory) following treatment with CPAP $[18,26]$. Thus, a variable change in loop gain with CPAP therapy or with drugs could be a potential mechanism of the observed variability in the return of events with CPAP withdrawal.

In the real world and in this study, all these factors contribute, alone and in combination, to the level of severity and/or development of sleep apnoea, not only as the patient presents and responds to short-term interventions but also with chronic CPAP therapy. Today, however, these elements can be measured using CPAP to clamp the airway pressure and initiate interventions to collect values related to muscle recruitment, arousals and loop gain [22].

There are always parts and pieces left over to consider. Longitudinal studies directed at the development of sleep apnoeas over time are few and the collected traits are more clinical (obesity, hypertension, etc.) than 
mechanistic ( $P_{\text {crit, }}$ loop gain, etc.). There is an underlying biology, which remains elusive. The primary molecular domains associated with sympathetic activity, oxidative stress and inflammation are present in some, but not all, cross-sectional studies [27]. Genetic studies have been underpowered, and causal versus consequential genetic effectors have not been distinguished in these studies [28], so there appears to be no clear path, leaving much room for inventive designs and original thought.

This paper then is not only a description of an effect but a blueprint for using CPAP withdrawal as a tool. For the stated purposes of the authors, this effect, for an individual, was unpredictable, using ODI. Using other designs and outcome measures, perhaps one can begin to use the stopping of CPAP, in the words of Rossi et al. [9], "equivalent to the average holiday", as a model for uncovering the reestablishment of OSA in humans.

\section{References}

Dempsey JA, Veasey SC, Morgan BJ, et al. Pathophysiology of sleep apnea. Physiol Rev 2010; 90: 47-112.

2 Bonsignore MR, Esquinas C, Barcelo A, et al. Metabolic syndrome, insulin resistance and sleepiness in real-life obstructive sleep apnoea. Eur Respir J 2012; 39: 1136-1143.

3 Kasasbeh E, Chi DS, Krishnaswamy G. Inflammatory aspects of sleep apnea and their cardiovascular consequences. South Med J 2006; 99: 58-67.

4 Kent BD, Garvey JF, Ryan S, et al. Severity of obstructive sleep apnoea predicts coronary artery plaque burden: a coronary computed tomographic angiography study. Eur Respir J 2013; 42: 1263-1270.

5 Almendros I, Montserrat JM, Ramirez J, et al. Intermittent hypoxia enhances cancer progression in a mouse model of sleep apnoea. Eur Respir J 2012; 39: 215-217.

6 Martinez-Garcia MA, Campos-Rodriguez F, Farre R. Sleep apnoea and cancer: current insights and future perspectives. Eur Respir J 2012; 40: 1315-1317.

7 Strohl KP, Cherniack NS, Gothe B. Physiologic basis of therapy for sleep apnea. Am Rev Respir Dis 1986; 134: 791-802.

8 Schwab RJ, Badr SM, Epstein LJ, et al. An official American Thoracic Society statement: continuous positive airway pressure adherence tracking systems. The optimal monitoring strategies and outcome measures in adults. Am J Respir Crit Care Med 2013; 188: 613-620.

9 Rossi VA, Schwarz EI, Bloch KE, et al. Is continuous positive airway pressure necessarily an everyday therapy in patients with obstructive sleep apnoea? Eur Respir J 2014; 43: 1387-1393.

10 Strohl KP, Redline S. Recognition of obstructive sleep apnea. Am J Respir Crit Care Med 1996; 154: 279-289.

11 White DP, Younes MK. Obstructive sleep apnea. Compr Physiol 2012; 2: 2541-2594.

12 Gold AR, Schwartz AR. The pharyngeal critical pressure. The whys and hows of using nasal continuous positive airway pressure diagnostically. Chest 1996; 110: 1077-1088.

13 Wellman A, Eckert DJ, Jordan AS, et al. A method for measuring and modeling the physiological traits causing obstructive sleep apnea. J Appl Physiol 2011; 110: 1627-1637.

14 Wellman A, Edwards BA, Sands SA, et al. A simplified method for determining phenotypic traits in patients with obstructive sleep apnea. J Appl Physiol 2013; 114: 911-922.

15 Kimoff RJ, Sforza E, Champagne V, et al. Upper airway sensation in snoring and obstructive sleep apnea. Am J Respir Crit Care Med 2001; 164: 250-255.

16 Grippo A, Carrai R, Romagnoli I, et al. Blunted respiratory-related evoked potential in awake obstructive sleep apnoea subjects: a NEP technique study. Clin Neurophysiol 2011; 122: 1562-1568.

17 Ratnavadivel R, Stadler D, Windler S, et al. Upper airway function and arousability to ventilatory challenge in slow wave versus stage 2 sleep in obstructive sleep apnoea. Thorax 2010; 65: 107-112.

18 Loewen A, Ostrowski M, Laprairie J, et al. Determinants of ventilatory instability in obstructive sleep apnea: inherent or acquired? Sleep 2009; 32: 1355-1365.

19 Strohl KP, Butler JP, Malhotra A. Mechanical properties of the upper airway. Compr Physiol 2012; 2: 1853-1872.

20 Eckert DJ, Younes MK. Arousal from sleep: implications for obstructive sleep apnea pathogenesis and treatment. J Appl Physiol 2014; 116: 302-313.

21 Younes M. Role of arousals in the pathogenesis of obstructive sleep apnea. Am J Respir Crit Care Med 2004; 169: 623-633.

22 Eckert DJ, White DP, Jordan AS, et al. Defining phenotypic causes of obstructive sleep apnea. Identification of novel therapeutic targets. Am J Respir Crit Care Med 2013; 188: 996-1004.

23 Wellman A, Malhotra A, Jordan AS, et al. Effect of oxygen in obstructive sleep apnea: Role of loop gain. Respir Physiol Neurobiol 2008; 162: 144-151.

24 Fan JL, Burgess KR, Thomas KN, et al. Influence of indomethacin on ventilatory and cerebrovascular responsiveness to $\mathrm{CO} 2$ and breathing stability: The influence of PCO2 gradients. Am J Physiol Regul Integr Comp Physiol 2010; 298: R1648-R1658.

25 Edwards BA, Sands SA, Feeney C, et al. Continuous positive airway pressure reduces loop gain and resolves periodic central apneas in the lamb. Respir Physiol Neurobiol 2009; 168: 239-249.

26 Salloum A, Rowley JA, Mateika JH, et al. Increased propensity for central apnea in patients with obstructive sleep apnea: effect of nasal continuous positive airway pressure. Am J Respir Crit Care Med 2010; 181: 189-193.

27 Arnardottir ES, Mackiewicz M, Gislason T, et al. Molecular signatures of obstructive sleep apnea in adults: a review and perspective. Sleep 2009; 32: 447-470.

28 Varvarigou V, Dahabreh IJ, Malhotra A, et al. A review of genetic association studies of obstructive sleep apnea: field synopsis and meta-analysis. Sleep 2011; 34: 1461-1468. 